\title{
Infestação de Aetalion reticulatum (Linnaeus) (Hemiptera: Auchenorrhyncha: Aethalionidae) em Plantas de Euterpe oleracea Martius (Arecaceae) no Estado do Acre
}

\author{
Rodrigo Souza Santos ${ }^{\circledR}{ }^{\bowtie}$, Antonio José Creão-Duarte \& Aureny Maria Pereira Lunz ${ }^{1}$
}

1. Embrapa Acre, e-mail: rodrigo.s.santos@embrapa.br (Autor para correspondência ${ }^{\varpi}$ ), aureny.lunz@embrapa.br. 2. Universidade Federal da Paraíba (UFPB), e-mail: creaoduarte@yahoo.com.br.

\section{EntomoBrasilis 8 (1): 69-73 (2015)}

Resumo. O açaizeiro (Euterpe oleracea Martius) é uma palmeira nativa da várzea da região amazônica e pode ser indicada como a espécie de maior valor econômico do gênero. Dos seus frutos é extraída a polpa, largamente consumida, de variadas formas, pela população da região Norte do Brasil. Com a expansão da área plantada, muitos fatores podem comprometer a produção e limitar o cultivo, destacando-se a ocorrência de insetospraga que causam prejuízos à produção. Este relato configura o primeiro registro de ocorrência da cigarrinha Aetalion reticulatum (Hemiptera: Auchenorrhyncha: Aethalionidae) em plantas de E. oleracea. A ocorrência deste inseto associado ao açaizeiro, em Rio Branco, Acre, aumenta a lista de hospedeiros desta cigarrinha no Brasil e reforça a necessidade de estudos de dinâmica populacional, levantamento de inimigos naturais, níveis de dano e métodos de controle, a fim de antecipar possíveis surtos populacionais desta praga em plantios comerciais de açaí no Estado.

Palavras-Chave: Amazônia; Arecaceae; cigarrinha; fitófago; surto de insetos.

\section{Infestation of Aetalion reticulatum (Linnaeus) (Hemiptera: Aethalionidae) in Plants of Euterpe oleracea Martius (Arecaceae) in Acre State, Brazil}

Abstract. The açai palm (Euterpe oleracea Martius) is a native palm tree from the Amazon region floodplains and may be indicated as the most economically profitable species of this genus. Its fruit pulp is extracted and widely consumed by the population of northern Brazil in several ways. With the expansion of the planted area many factors may affect the production and limit the cultivation, highlighting the occurrence of insect pests that cause losses in production. This report presents the first occurrence of the leafhopper Aetalion reticulatum (Linnaeus) (Hemiptera: Auchenorrhyncha: Aethalionidae) in E. oleracea plants. The occurrence of this insect associated with açai palms in Rio Branco, Acre, increases the list of hosts for this leafhopper in Brazil and reinforces the need for studies of population dynamics, survey of natural enemies, levels of damage and control methods in order to anticipate population outbreaks of this pest in commercial plantations of açai palms in the State.

Keywords: Amazon; Arecaceae; insect outbreak; leafhopper; phytophagous.

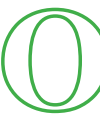
açaizeiro (Euterpe oleracea Martius) é conhecido por diversos nomes, tais como: açaí, açaí-do-pará, açaído-baixo-amazonas, açaí-de-touceira, açaí-da-várzea, juçara, juçara-de-touceira e açaí verdadeiro. É uma palmeira nativa da várzea da região amazônica e pode ser indicada como a espécie de maior valor econômico do gênero Euterpe, dentre as dez registradas no Brasil e as sete que ocorrem na Amazônia (Oliveira et al. 2002). Esta espécie está presente em toda a extensão do estuário amazônico, distribuída no baixo Amazonas, Maranhão, Tocantins e Amapá (SouzA et al. 1996), além de países da América do Sul (Venezuela, Colômbia, Equador, Suriname e Guiana) e da América Central (Panamá) (Nogueira 2005).

O Brasil se posiciona como o maior produtor, consumidor e exportador de açaí (Menezes 2005), de cujos frutos é extraída a polpa utilizada na preparação de suco, muito consumido pela população da região Norte do país (Oliveira et al. 2002; Hoмma et al. 2006). Além do suco, a polpa de açaí é largamente usada na produção industrial ou artesanal de sorvetes, geleias, compotas e licores (NASCIMENTO 2008), podendo, ainda, ser extraído o corante antocianina (Номмa et al. 2006). Do seu estipe é extraído o palmito, que possui excelente aceitação tanto no mercado nacional quanto internacional (Souza \& Lemos 2004). Os principais Estados produtores brasileiros são Pará, Maranhão e Acre, sendo o Pará o responsável por $89 \%$ da quantidade produzida no país (Fundação Banco do Brasil 2010). Com o aumento do consumo, principalmente visando às demandas internacionais, o estado do Acre almeja o incremento do plantio em larga escala (PoRTAL DA AMAZÔNIA 2013).

Desta forma, com a expansão da área plantada, muitos fatores podem comprometer a produção e limitar o cultivo. Dentre eles, a ocorrência de insetos-praga ocupa lugar de destaque, pois são relatadas diversas espécies de insetos atacando o açaizeiro (Oliveira et al. 2002), particularmente na fase de viveiro (SouZA 2002; SouZA \& LEMOS 2004, 2005). Os problemas fitossanitários causados por estes organismos têm surgido com maior evidência e aumentado consideravelmente, tornando-se preocupante em função dos prejuízos que vêm causando ao açaizeiro (SouzA \& LEMOS 2004, 2005).

Diversos insetos são capazes de atacar o açaizeiro desde a fase de sementeira até a fase adulta. Portanto, torna-se necessário que sejam conhecidas as pragas que atacam esta palmeira, pois este conhecimento poderá ser utilizado como mais um parâmetro a ser incorporado no processo de melhoramento genético do açaizeiro, permitindo que se façam recomendações seguras de controle dos principais organismos-praga (SouZA \& LEMOS 2004).

Atualmente, um complexo de insetos-praga está associado a $E$. oleracea, ocasionando injúrias, de acordo com seus níveis de infestação, tais como: os pulgões [Cerataphis lataniae Boisudval 
T e Cerataphis brasiliensis (Hempel)]; as moscas-brancas [Aleurothrixusfloccosus(Maskell)eAleurodicuscoccois(Curtis)]; as formigas cortadeiras (Atta spp.), os besouros [Rhynchophorus palmarum Linnaeus, Rhinostomus barbirostris (Fabricius), Pachymerus nucleorum (Fabricius), Hemisphaerota tristis (Boheman), Dynamis borassi Fabricius, Xylosandrus compactus (Eichhoff), Xyleborus ferrugineus (Fabricius), Macraspis pseudochrysis Landin, Metamasius hemipterus (Linnaeus), Foveolus maculatus O'brien e Coccotrypes sp.]; as cochonilhas [Mytilococcus (Lepidosaphis) beckii (Newman) e Aspidiotus destructor Signoret]; os gafanhotos [Tropidacris collaris (Stoll) e Eutropidacris cristata Linnaeus] e as mariposas [Synale hylaspes (Cramer), Opsiphanes invirae (Huebner), Eupalamides cyparissias Fabricius e Brassolis sophorae (Linnaeus)] (ZoRZENON \& Bergmann 1995; Souza et al. 2000; Gallo et al. 2002; Oliveira et al. 2002; Souza 2002; Souza \& Lemos 2004, 2005; Beserra et al. 2006; Jordão \& Silva 2006; BERnardino 2007; NASCIMENTO 2008; Lunz et al. 2011; Jesus-Barros et al. 2013).

Em março de 2014 foram observadas colônias de cigarrinhas em plantas de E. oleracea, em plantio localizado no Campo Experimental da Embrapa Acre (1001'26"S; 6704'39”W), município de Rio Branco. As colônias se encontravam nas ráquilas de inflorescências e infrutescências, e a infestação estava distribuída de forma não homogênea no talhão (reboleira). Foram realizadas três inspeções na área, sendo constatado, em cada vistoria, um aumento populacional da cigarrinha, bem como do número de plantas infestadas.

O talhão, denominado "Área de Produção de Sementes (APS)", possui área de 1,0 ha, com 400 plantas de quatro anos de idade, altura média de $3,5 \mathrm{~m}$ e cultivadas no espaçamento de 5,0 × $5,0 \mathrm{~m}$. A plantas são oriundas de sementes de indivíduos selecionados da população geneticamente melhorada da cultivar BRS-Pará. Os tratos culturais foram efetuados de acordo com as recomendações técnicas para o açaizeiro e a área sofreu uma aplicação de óleo mineral em dezembro de 2013, visando o controle de pulgões.

Insetos adultos foram coletados manualmente nas ráquilas das inflorescências e infrutescências, acondicionados em frascos contendo álcool etílico a 70\%, devidamente identificados, e levados até o Laboratório de Entomologia da Embrapa Acre, onde foram triados, montados e etiquetados. Os espécimes foram enviados para identificação e chegou-se à espécie Aetalion reticulatum (Linnaeus) (Hemiptera: Aethalionidae) (Figura 1), espécie conhecida como cigarrinha-das-fruteiras, cigarrinhados-pomares ou cigarrinha-do-pedúnculo (GALLo et al. 2002). Foram analisados 137 espécimes, sendo 70 machos, 20 fêmeas e 47 ninfas, os quais foram integrados ao acervo da Coleção Entomológica do Departamento de Sistemática e Ecologia da Universidade Federal da Paraíba (UFPB).

A cigarrinha-das-fruteiras é um inseto fitófago, cujos adultos medem em torno de $10 \mathrm{~mm}$ de comprimento, de coloração marrom ferrugínea, com venação das asas salientes e esverdeadas. As posturas podem conter até 100 ovos, colocados em massas, na superfície dos ramos das plantas hospedeiras. Os ovos demoram cerca de 30 dias para eclodirem; durante este tempo, a fêmea permanece junto à postura, protegendo-a. Permanecem nesta fase durante 45 dias, completando seu ciclo em torno de 110 dias, com três gerações ao ano (SANTANA et al. 2005). Tanto os adultos quanto as ninfas sugam continuamente a seiva da planta, prejudicando o desenvolvimento de frutos e brotações, podendo, em altas infestações, matar o hospedeiro (GALlo et al. 2002). Os adultos vivem cerca de dois meses (VIEIRA et al. 2007; RANDo \& Lima 2010) e possuem relações comensais e/ou simbióticas com outros insetos, tais como: formigas do gênero Camponotus, a vespa Synoeca septentrionalis Richard (Hymenoptera: Vespidae) e abelhas-sem-ferrão (Meliponini), principalmente Trigona, as quais coletam as secreções açucaradas (honeydew), ricas em nutrientes, e protegem a colônia contra insetos invasores (RAMONI-PERAZZi et al. 2006; OdA et al. 2009; BARÔNIO et al. 2012). Foi observada a presença de abelhas-sem-ferrão e formigas do gênero Camponotus associadas às cigarrinhas nas plantas infestadas, mas não simultaneamente.

Nas infrutescências infestadas pela cigarrinha, os frutos apresentaram intensa formação de fumagina (Figura 2) devido à constante exsudação de honeydew pelos adultos e ninfas presentes. A presença de A. reticulatum também foi observada em inflorescências, o que indica que o ataque do inseto precede a

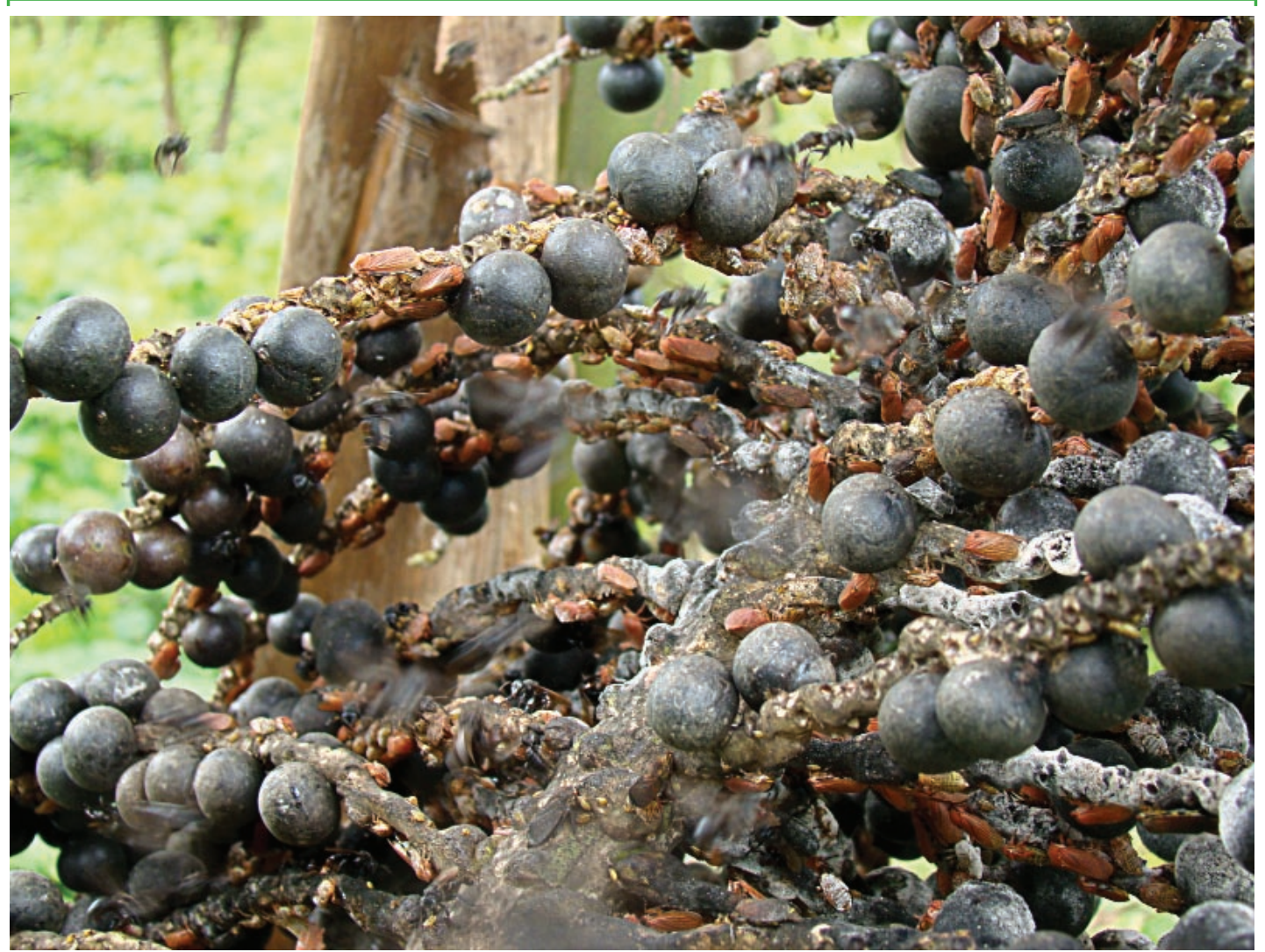

Figura 1. Colônia de Aetalion reticulatum (Hemiptera: Aethalionidae) em ráquilas de Euterpe oleracea, no município de Rio Branco, Acre. 


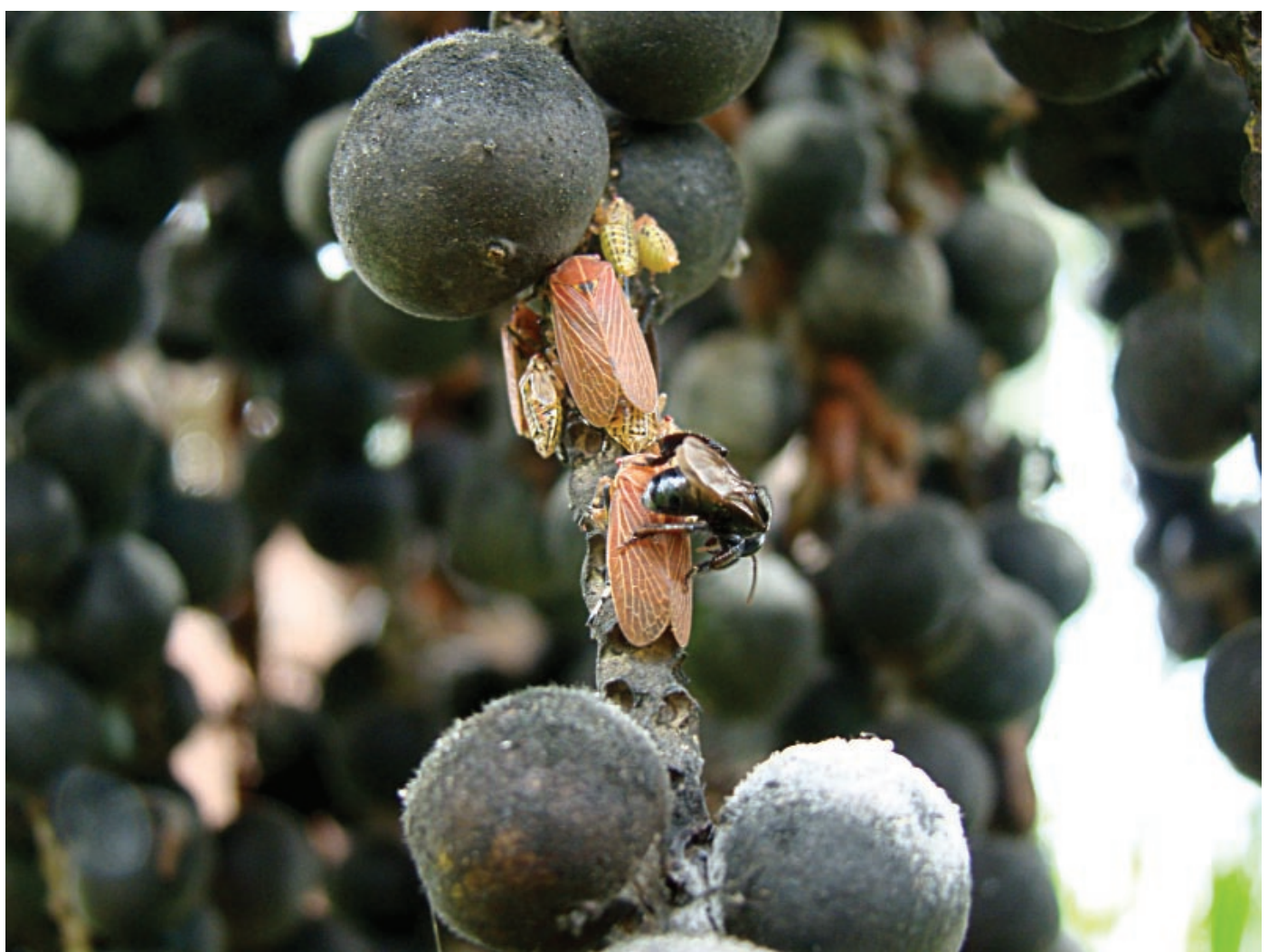

Figura 2. Desenvolvimento de fumagina na superfície de frutos de Euterpe oleracea.

formação dos frutos em E. oleracea. Desta forma, altas infestações de $A$. reticulatum podem promover atraso no desenvolvimento e queda de frutos, pela contínua sucção de seiva. Além disso, frutos cobertos por fumagina demandam maiores cuidados na higienização antes de seu processamento.

A espécie $A$. reticulatum é amplamente distribuída, ocorrendo desde a América Central à América do Sul (MARQues 1928). No Brasil, sua ocorrência é relatada nos seguintes Estados: AM, AP, BA, CE, ES, DF, GO, MG, PA, PR, RJ, RS, SC e SP (MARQUES 1928; SANTANA et al. 2005; JordÃo \& Silva 2006; BARÔNIO et al. 2012). É um inseto polífago, que se alimente de seiva das folhas e ramos de plantas medicinais, nativas, leguminosas, ornamentais, invasoras, florestais e, principalmente, frutíferas (Silva et al. 1968; Costa 1986; Gallo et al. 2002; Santana et al. 2005; CARVAlHo 2007; AzEvedo et al. 2008; Ото et al. 2009; RANDo \& Lima 2010; Menezes et al. 2012; CAMILo et al. 2013), mas que ainda não havia sido relatado associado ao açaizeiro.

No que tange ao controle biológico, são relatados como inimigos naturais deste aetalionídeo os parasitoides de ovos: Gonatocerus (Gahanopsis) aethalionis (Oglobin) (Hymenoptera: Mymaridae); Pterygogramma (Abbelloides) marquesi (Brèthes), Lathromerella sp. e Uscanopsis sp. (Hymenoptera: Trichogrammatidae) e de ninfas: Metablastothrix (Mycrotherys) claripennis (Compere) (Hymenoptera: Encyrtidae) (Silva et al. 1968; SugonjaEv \& TRIAPITZYN 1988; Viggiani 1992; CARVALHO 2007; TriaptizYN et al. 2010). Ainda, é relatada a associação do tripes Aulacothrips dictyotus Hood (Thysanoptera: Heterothripidae), como ectoparasita de $A$. reticulatum, encontrado debaixo das asas de adultos ou sob os rudimentos alares das ninfas (Izzo et al. 2002). Em relação a outros métodos de controle, não há registros de produtos fitossanitários registrados para o controle desta cigarrinha em açaizeiro. Entretanto, inseticidas fosforados, carbamatos ou reguladores de crescimento são recomendados, como opções de controle químico desta cigarrinha em outras culturas (GALLO et al. 2002). Como método alternativo de controle, MARQUES (1928) recomenda a utilização de emulsão de água (4 L), sabão (500 g) e querosene ( 8 L), diluída em 8 a 10 partes de água e aplicada diretamente sobre as ninfas e adultos, com intervalo de 15 a 20 dias, se necessário. Um método de controle agroecológico, proposto por Silva et al. (2007), é a utilização de extrato aquoso de folhas ou raízes de Piper aduncum L. (pimenta-de-macaco), que apresenta atividade inseticida sobre adultos de Aetalion $\mathrm{sp.}$

Este relato configura o primeiro registro de ocorrência de $A$. reticulatum, associada às plantas de E. oleracea no estado do Acre. A ocorrência de infestações desta cigarrinha, em altos níveis populacionais, pode causar prejuízo no desenvolvimento e queda dos frutos pela redução da quantidade de nutrientes, ocasionada pela constante alimentação do inseto. Estudos de dinâmica populacional, levantamento de inimigos naturais, níveis de dano e métodos de controle são recomendados, a fim de traçar estratégias de monitoramento e controle de possíveis surtos populacionais desta praga em plantios comerciais de açaí no Estado.

\section{AGRADECIMENTOS}

À mestranda Luciana Maira de Sales Pereira (Universidade Federal do Acre - UFAC), pela revisão do texto e Abstract.

\section{REFERÊNCIAS}

Azevedo, R.L., C.A.L. Carvalho \& O.M. Marques, 2008. Insetos associados à cultura do feijão na região do recôncavo da Bahia, Brasil. Revista Caatinga, 21: 83-88.

Barônio, G.J., A.C.V. Pires \& C. Aoki, 2012. Trigona branneri (Hymenoptera: Apidae) as a collector of honeydew from Aethalion reticulatum (Hemiptera: Aethalionidae) on Bauhinia forficata (Fabaceae: Caesalpinoideae) in a Brazilian savanna. Sociobiology, 59: 407-414.

Bernardino, A.S., 2007. Flutuação populacional da brocada-coroa-foliar Eupalamides cyparissias (Lepidoptera: Castniidae) em plantios de dendê (Elaeis guineensis) no estado do Pará. Dissertação (Mestrado em Entomologia). Universidade Federal de Viçosa. 37 p.

Beserra, P., G. Couturier \& M.S.P. Oliveira, 2006. Cultivated açai palm (Euterpe oleracea) and associated weevils: Foveolus maculatus and Dynamis borassi (Coleoptera: Dryophthoridae). Palms, 50: 120-122.

Camilo, S.S., M.A. Soares, S.L. Assis Júnior \& E.S. Pereira, 2013. Infestação de Aethalion reticulatum (L., 1767) (Hemiptera: Aethalionidae) em plantas de Dictyoloma vandellianum Adr. 
Juss. (Rutaceae). MG. Biota, 5: 4-12.

Carvalho, R.C.d'L., 2007. Interações entre A. reticulatum (Aetalionidae: Hemiptera) e seus mutualistas: Meliponini (Apinae: Apidae: Hymenoptera) e Camponotus spp. (Formicidae: Hymenoptera). Dissertação (Mestrado em Ciências: Entomologia). Faculdade de Filosofia, Ciências e Letras de Ribeirão Preto. 117 p.

Costa, E.C., 1986. Artrópodes associados à Bracatinga (Mimosa scrabella Benth.). Tese (Doutorado em Ciências Florestais) Universidade Federal do Paraná. 271 p.

Fundação Banco do Brasil. 2010. Fruticultura - Açaí. Desenvolvimento Regional Sustentável. Brasília: Fundação Banco do Brasil. 52p. (Série cadernos de propostas para atuação em cadeias produtivas, 2). Disponível em: <http:// www.bb.com.br/docs/pub/inst/dwn/Vol2FruticAcai.pdf $>$. Acesso em: 19 mar. 2015.

Gallo, D., O. Nakano, S. Silveira-Neto, R.P.L. Carvalho, G.C. Batista, E. Berti Filho, J.R.P. Parrra, R.A. Zucchi, S.B. Alves, J.D. Vendramim, L.C. Marchini, J.R.S. Lopes \& C. Omoto, 2002. Entomologia Agrícola. Piracicaba: FEALQ, 920p.

Homma, A.K.O., O.L. Nogueira, A.J.E.A. Menezes, J.E.U. Carvalho, C.M.L. Nicoli \& G.B. Matos, 2006. Açaí: novos desafios e tendências. Amazônia: Ciência \& Desenvolvimento, 1: 7-23.

Izzo, T.J., S.M.J. Pinent \& L.A. Mound, 2002. Aulacothrips dictyotus (Thysanoptera: Heterothripidae): the first ectoparasitic thrips (Thysanoptera). Florida Entomologist, 85: 281-283.

Jesus-Barros, C.R., L.S. Freiras \& P.C. Grossi, 2013. Report of Macraspis pseudochrysis Landin, 1956 (Coleoptera: Scarabaeidae: Rutelinae) on açai palms (Euterpe oleracea Mart.) in floodplains in the state of Amapá, Brazil. Biota Neotropica, 13: 380-382.

Jordão, A.L. \& R.A. Silva, 2006. Guia de pragas agrícolas para o manejo integrado no estado do Amapá. Ribeirão Preto: Holos, $183 \mathrm{p}$.

Lunz, A.M., S.M.N. Lazzari, R.C. Zonta-de-Carvalho, M.S.P. Oliveira \& L.A. Souza, 2011. Cerataphis brasiliensis (Hempel) (Hemiptera: Aphididae) em quatro espécies de palmáceas na Amazônia: ocorrência e considerações taxonômicas. Acta Amazônica, 41: 593-596.

Marques, L. A. de A., 1928. Cigarrinha nociva a várias espécies vegetais: biologia do membracídeo Aethalium reticulatum (L.). Instituto Biológico de Defesa Agrícola e Animal, Boletim 6, $27 \mathrm{p}$.

Menezes, C.W.G., M.A. Soares, S.L. Assis Júnior, A.J. Fonseca, E.M. Pires \& J.B. Santos, 2012. Novos insetos sugadores (Hemiptera) atacando Eucalyptus cloeziana (Myrtaceae) em Minas Gerais, Brasil. Entomobrasilis, 3: 246-248.

Menezes, E.M.S., 2005. Efeito da alta pressão hidrostática em polpa de açaí pré-congelada (Euterpe oleracea, Mart.). Dissertação (Mestrado em Ciência e Tecnologia de Alimentos) - Universidade Federal Rural do Rio de Janeiro. 83 p.

Nascimento, W.M.O., 2008. Açaí: Euterpe oleracea Mart. Informativo Técnico: Rede de Sementes da Amazônia, 1: 1-2.

Nogueira, O.L., 2005. Introdução e importância econômica, p 1113. In: Nogueira, O.L., F.J.C. Figueiredo \& A.A. Müller (Org.). Sistema de produção 4: Açaí. Belém: Embrapa Amazônia Oriental, $137 \mathrm{p}$.

Oda, F.H., C. Aoki, T.M. Oda, R.A. Silva \& M.F. Felismino, 2009. Interação entre Trigona hyalinata (Lepeletier, 1836) (Hymenoptera: Apidae) e Aethalion reticulatum Linnaeus, 1767 (Hemiptera: Aethalionidae) em Clitoria fairschildiana Howard (Papilionoideae). Entomobrasilis, 2: 58-60.

Oliveira, M.S.P., J.E.U. Carvalho, W.M.O. Nascimento \& C.H. Müller, 2002. Cultivo do açaizeiro para produção de frutos. Belém: Embrapa Amazônia Oriental (Circular Técnica, 26), $17 \mathrm{p}$.

Oto, M.M., A.M. Sakakibara, M.J.S. Santana, A.J.A. Carvalho \& M.L. Coutinho, 2009. Espécies de Membracoidea (Insecta: Hemiptera) associadas a erva-de-passarinho Struthantus marginatus (Desr.) Blume (Loranthaceae) em Cruz das Almas, Bahia. Magistra, 21: 219-221.

Portal da Amazônia, 2013. Acre vai exportar açaí em pó para Oceania e Ásia em 2014. Disponível em: <http://www. revistaamazonia.com.br/estados/acre/4110-acre-vaiexportar-acai-em-po-para-oceania-e-asia-em-2014> Acesso em: 23 out. 2014.

Ramoni-Perazzi, P., G. Bianchi-Pérez \& G. Bianchi-Ballesteros, 2006. Primer registro de asociácion entre Aetalion reticulatum (Linné) (Hemiptera: Aetalionidae) y Synoeca septentrionalis Richards (Hymenoptera: Vespidae). Entomotropica, 21: 129132.

Rando, J.S.S. \& C.B. Lima, 2010. Detecção de Aethalion reticulatum (L., 1717) (Hemiptera: Aethalionidae) em alfavaca-cravo (Ocimum gratissimum L.) e observações sobre sua ocorrência. Revista Brasileira de Plantas Medicinais, 12: 239-242.

Santana, D.L.Q., C.A. Ferreira, E.G. Martins \& H.D. Silva, 2005. Ocorrência de Aethalion reticulatum (Linnaeus, 1767) (Hemiptera: Aethalionidae) em Grevillea robusta. Boletim de Pesquisa Florestal, 50: 109-115.

Silva, A.G.d'A., C.R. Gonçalves, D.M. Galvão, A.J.L. Gonçalves, J. Gomes, M.N. Silva \& L. Simoni, 1968. Quarto catálogo dos insetos que vivem nas plantas do Brasil: seus parasitos e predadores. Rio de Janeiro, Ministério da Agricultura. 622 p.

Silva, W.C., J.D’Arc Ribeiro, H.E.M. Souza \& R.S. Côrrea, 2007. Atividade inseticida de Piper aduncum L. (Piperaceae) sobre Aetalion sp. (Hemiptera: Aetalionidae), praga de importância econômica no Amazonas. Acta Amazonica, 37: 293-298.

Souza, A.G.C., N.R. Souza, S.E.L. Silva, C.D.M. Nunes, A.C. Canto \& L.A.A. Cruz, 1996. Fruteiras da Amazônia. Brasília: Embrapa-CPAA, 204p.

Souza, L.A. \& W.P. Lemos, 2004. Prospecção de insetos associados ao açaizeiro (Euterpe oleracea Mart.) em viveiro e proposições de controle. Revista de Ciências Agrárias, 42: 231-241.

Souza, L.A. \& W.P. Lemos, 2005. Pragas e Métodos de Controle. p. 63-81. In: Nogueira, O.L., F.J.C. Figueiredo \& A.A. Müller (Org.). Sistemas de Produção 4: Açaí. $1^{\mathrm{a}}$ ed. Belém: Embrapa Amazônia Oriental, 137 p.

Souza, L.A., 2002. Insetos pragas em acessos de açaizeiro em viveiro. Belém: Embrapa Amazônia Oriental (Comunicado Técnico, 75), 5p.

Souza, L.A., P. Celestino Filho \& A.B. Silva, 200o. Principais pragas do dendezeiro e seu controle, p. 276-334. In: I.J.M. Viégas \& A.A. Müller (eds.). A cultura do dendezeiro na Amazônia brasileira. Belém: Embrapa Amazônia Oriental; Manaus: Embrapa Amazônia Ocidental. 347p.

Sugonjaev, E.S. \& V.A. Triapitizyn, 1988. Chalcids of the genus Metablastothrix Sugonjaev (Hymenoptera, Chalcidoidea) and peculiarities of their distribution in North America and Eurasia. Entomologicheskoe Obozrenie, 67: 182-187.

Triaptizyn, S.V., J.T. Huber, G.A. Logarzo, V.V. Berezovskiy \& D.A. Aquino, 2010. Review of Gonatocerus (Hymenoptera: Mymaridae) in the Neotropical region, with description of eleven new species. Zootaxa, 2456: 1-243.

Vieira, C.U., C.M. Rodovalho, L.O. Almeida, A.C.S. Siquieroli \& A.M. Bonetti, 2007. Interação entre Trigona spinipes Fabricius, 1793 (Hymenoptera: Apidae) e Aethalion reticulatum Linnaeus, 1767 (Hemiptera: Aethalionidae) em Mangifera indica (Anacardiaceae). Bioscience Journal, Supplement 1, 23: 10-13.

Viggiani, G., 1992. New species of Trichogrammatidae (Hymenoptera: Chalcidoidea) from South America. Redia, 75: 253-265.

Zorzenon, F.J. \& E.C. Bergmann, 1995. Ocorrência de Xyleborus ferrugineous (Fabricius, 1801) (Coleoptera: Scolytidae) em frutos e sementes de duas espécies do gênero Euterpe. Revista de Agricultura, 70: 17-20. 
Recebido em: 18/o6/2014

Aceito em: 30/10/2014

$* * * * * * * * *$

Como citar este artigo:

Santos, R.S., A.J. Creão-Duarte \& A.M.P. Lunz, 2015. Infestação de Aetalion reticulatum (Linnaeus) (Hemiptera: Auchenorrhyncha: Aethalionidae) em Plantas de Euterpe oleracea Martius (Arecaceae) no Estado do Acre. EntomoBrasilis, 8 (1): 69-73.

Acessível em: $\underline{\text { doi:10.12741/ebrasilis.v8i1.450 }}$
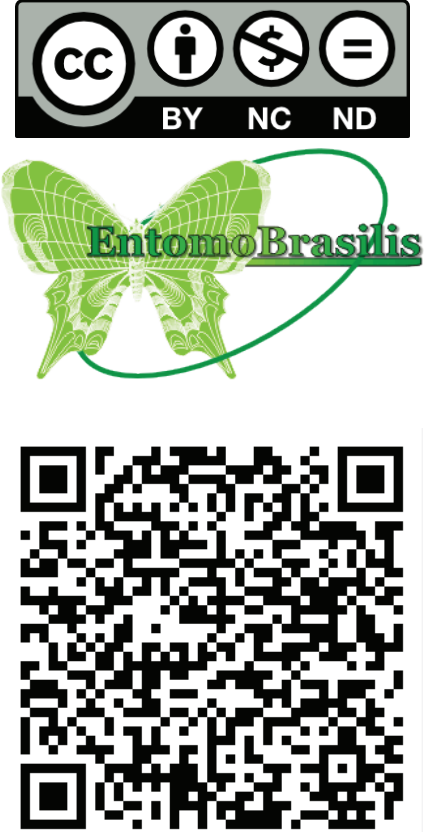